This item was submitted to Loughborough's Research Repository by the author.

Items in Figshare are protected by copyright, with all rights reserved, unless otherwise indicated.

\title{
Scheduling and control co-design of networked induction motor control systems
}

PLEASE CITE THE PUBLISHED VERSION

http://dx.doi.org/10.1109/ICInfA.2013.6720418

PUBLISHER

(C) IEEE

VERSION

VoR (Version of Record)

\section{PUBLISHER STATEMENT}

This work is made available according to the conditions of the Creative Commons Attribution-NonCommercialNoDerivatives 4.0 International (CC BY-NC-ND 4.0) licence. Full details of this licence are available at: https://creativecommons.org/licenses/by-nc-nd/4.0/

\section{LICENCE}

CC BY-NC-ND 4.0

\section{REPOSITORY RECORD}

Zhao, Dezong, Shangmin Zhang, Chunwen Li, and Richard Stobart. 2017. "Scheduling and Control Co-design of Networked Induction Motor Control Systems”. figshare. https://hdl.handle.net/2134/26515. 


\title{
Scheduling and Control Co-Design of Networked Induction Motor Control Systems
}

\author{
Dezong Zhao ${ }^{1}$, Member, IEEE, Shangmin Zhang ${ }^{2}$, Chunwen $\mathrm{Li}^{2}$, and Richard Stobart ${ }^{1}$, Member, IEEE
}

\begin{abstract}
This paper investigates the co-design of remote speed control and network scheduling for motion coordination of multiple induction motors through a shared communication network. An integrated feedback scheduling algorithm is designed to allocate the optimal sampling period and priority to each control loop to optimize the global performance of a networked control system (NCS), while satisfying the constraints of stability and schedulability. The rational gain of the network speed controllers is calculated using the Lyapunov theorem and online tuned by fuzzy logic to guarantee the robustness against complicated variations on the communication network. Furthermore, a state predictor is designed to compensate the time delay occurred in data transmission from the sensor to the controller, as a part of the networked controller. Simulation results are given to illustrate the effectiveness of the proposed control-and-scheduling co-design approach.
\end{abstract}

\section{INTRODUCTION}

The applications of NCSs have been an important trend in modern industry owing to the convenient remote operation and cost-effective installation. In such systems, spatially distributed sensors, actuators, and controllers share information through the network instead of complex wiring, resulting in flexible and open architecture. NCSs have been found applications in a broad range of areas such as mobile robots [1], unmanned aerial vehicles [2], and remote surgery [3]. Considering the common grounds that they are driven by electrical motors and communicate via network, such systems are called networked motion control systems (NMCSs) [4]. NMCSs are constructed on the basis of remote motion controller and local motor drivers, using network to realize transmission of control orders and motion states. NMCSs are hot research topics of NCSs and play important roles in factory automation. Most of the current NMCSs focus on networked DC motor control [5], for DC motor is an ideal networked control plant with linear model. Actually, induction motors play a dominant part in industrial applications for their merits of simple structure and high reliability. However, networked induction motor control is rather more complicated due to the nonlinear dynamics of induction motors. Networked induction motor control is a rather challenging research topic.

This work was supported by National Natural Science Foundation of China under the grant reference 61174068

${ }^{1}$ D. Zhao and R. Stobart are with Department of Aeronautical and Automotive Engineering, Loughborough University, Loughborough, Leicester, UK (e-mail: d.zhao2@lboro.ac.uk, r.k.stobart@lboro.ac.uk)

${ }^{2} \mathrm{~S}$. Zhang and $\mathrm{C}$. Li are with Department of Automation \& Tsinghua National Laboratory for Information Science and Technology, Tsinghua University, Beijing, China (e-mail: jiang.nan.bu.yi@163.com, lcw@mail.tsinghua.edu.cn)
New concepts of operation bring new notions in the control system, including the quality of service (QoS), link, and configuration. Time delay and packets dropout are the two most important issues to be concerned which would result in NCSs performance deterioration and potential system instability. It is particularly important in dealing with the two issues in designing networked motion controllers, such as gain scheduling and sampling period adaptation, for NMCSs are time critical due to their fast dynamics. The NCS control strategies can be grouped into two categories: stability analysis based methods [6] and system synthesis methods [7]. In stability analysis based methods, the NCS controllers are designed primarily with the assumption of no information lost, then analyze the system performance considering the network environment. The system synthesis methods are more practical, where the controller parameters and sampling periods are obtained with the consideration of communication constraints.

On the other hand, the overall performance of a multipleloop NCS depends on both of the control algorithm and scheduling algorithm. The traditional static scheduling methods cannot find the optimal solution of the NCS for the sampling period and priority of each loop are calculated offline [8]. Considering the tradeoff between the quality of service (QoS) of the network and the quality of control (QoC) of the NCS, the co-design of network controller and scheduling method is an efficient way [9]. In the co-design method, the scheduling algorithm updates the sampling period and priority of each loop online, such that the global optimization of the NCS is approached.

In this paper, an integrated feedback scheduling strategy is proposed, including the optimal bandwidth allocation scheme, online priority modification scheme, and adjacent cross coupling control structure. An optimization problem is formulated as minimizing the sum of the tracking error of each control loop, with the constraints of stability and available network bandwidth, to improve the speed synchronization performance of the NMCS. In designing the networked speed controller, its rational gain is calculated using the Lyapunov theorem and tuned online by fuzzy logic.

The paper is organized as following. After the introduction in section I, the system description is presented in section II. The networked speed controller is proposed in section III. The integrated feedback scheduling strategy is presented in section IV. The simulation results are stated in section V. Finally, the conclusions are summarized in section VI. 


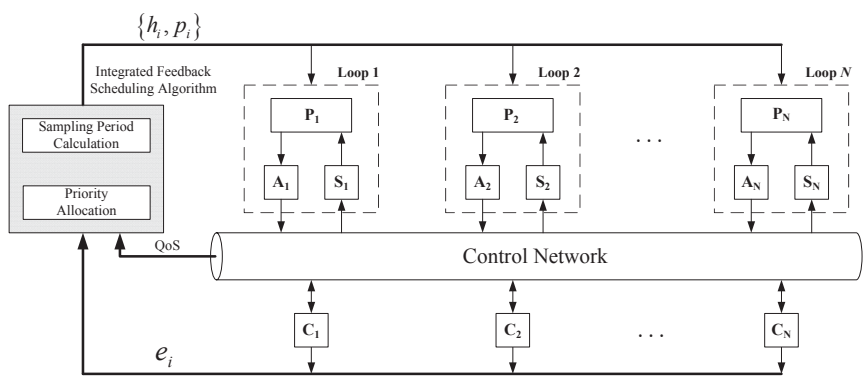

Fig. 1. Structure diagram of the investigated NMCS

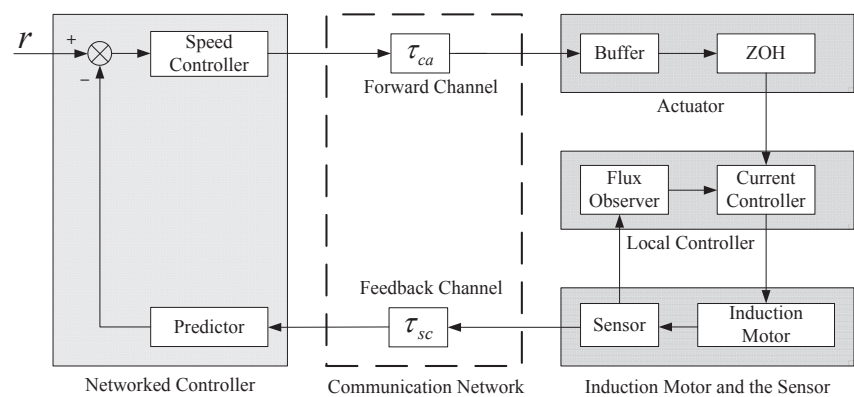

Fig. 2. Structure of a single control loop in the NMCS

\section{SYSTEM DESCRIPTION}

The structure diagram of the investigated NMCS is shown as Fig. 1 in more details, where $A_{i}, C_{i}, S_{i}$, and $P_{i}$ denote the actuator, controller, sensor, and plant in loop $i$, respectively. The bandwidth-limited control network is shared by $N$ control loops therein. In the NMCS, a priority-driven medium access control (MAC) protocol is employed, such as the DeviceNet. According to the non-preemptive scheduling standard, each loop is assigned with a unique priority. In loop $i$, the output speed of motor $P_{i}$ is sampled by $S_{i}$ with the sampling period of $h_{i}$, and sent to $C_{i}$ with the priority $p_{i}$. A computer or a node in the application layer behaves as the master node to perform the integrated feedback scheduling algorithm. In the decision making process, $h_{i}$ and $p_{i}$ are updated according to the QoS and the feedback speed of all loops.

In our co-design methodology, the following assumptions are made: (1) The sensor is time driven; (2) The controller and the actuator are event driven; and (3) The data sampled in one period can be encapsulated and transmitted in one packet.

As shown in Fig. 2, the components of each control loop can be grouped into five modules: (1) the induction motor and the sensor; (2) the communication network; (3) the networked controller; (4) the actuator; and (5) the local controller, which are described in the following subsections, respectively.

\section{A. Induction Motor and the Sensor}

The dynamics of a three-phase squirrel induction motor in the stator fixed $\alpha-\beta$ reference frame is described as the following differential equations [10]:

$$
\begin{aligned}
& \dot{i}_{\alpha s}=-\gamma i_{\alpha s}+\alpha \beta \psi_{\alpha r}+n_{p} \beta \omega \psi_{\beta r}+u_{\alpha s} /\left(\sigma L_{s}\right), \\
& \dot{i}_{\beta s}=-\gamma i_{\beta s}+\alpha \beta \psi_{\beta r}-n_{p} \beta \omega \psi_{\alpha r}+u_{\beta s} /\left(\sigma L_{s}\right), \\
& \dot{\psi}_{\alpha r}=\alpha M i_{\alpha s}-\alpha \psi_{\alpha r}-n_{p} \omega \psi_{\beta r}, \\
& \dot{\psi}_{\beta r}=\alpha M i_{\beta s}-\alpha \psi_{\beta r}+n_{p} \omega \psi_{\alpha r}, \\
& \dot{\omega}=\mu\left(\psi_{\alpha r} i_{\beta s}-\psi_{\beta r} i_{\alpha s}\right)-\left(T_{L}+K_{f} \omega\right) / J .
\end{aligned}
$$

where the two-dimensional vectors $i_{s}=\left[\begin{array}{ll}i_{\alpha s} & i_{\beta s}\end{array}\right]^{\mathrm{T}}, \psi_{r}=$ $\left[\begin{array}{ll}\psi_{\alpha r} & \psi_{\beta r}\end{array}\right]^{\mathrm{T}}$, and $u=\left[\begin{array}{ll}u_{\alpha s} & u_{\beta s}\end{array}\right]^{\mathrm{T}}$ are the stator currents, rotor fluxes, and stator voltages, respectively. $\omega$ is the mechanical rotor speed, $R_{S}$ and $R_{r}$ are the stator and rotor resistances, respectively; $L_{S}$ and $L_{r}$ are the stator and rotor self-inductances, respectively; $M$ is the stator-rotor mutual inductance, $T_{L}$ is the load torque, $K_{f}$ is the friction coefficient, $J$ is the motor-load moment of inertia, and $n_{p}$ is the number of pole pairs. Denote the leakage factor by $\sigma=1-M^{2} /\left(L_{S} L_{r}\right)$, the rotor time constant by $T_{r}=L_{r} / R_{r}$, and the other parameters by $\alpha=1 / T_{r}, \beta=M /\left(\sigma L_{S} L_{r}\right)$, $\gamma=M^{2} R_{r} /\left(\sigma L_{S} L_{r}^{2}\right)+R_{s} /\left(\sigma L_{s}\right)$, and $\mu=3 n_{p} M /\left(2 J L_{r}\right)$. The mechanical equation (1e) can be expressed in terms of the electromagnetic torque $T_{e}$ :

$$
T_{e}=J \dot{\omega}+K_{f} \omega+T_{L}
$$

The induction motor speed is measured by the sensor periodically, and be sent to the networked controller via the network together with its time stamp.

\section{B. Communication Network}

The network-induced delay consists of the sensor-tocontroller delay $\tau_{s c}$ and the controller-to-actuator delay $\tau_{c a}$, and can be lumped together as $\tau=\tau_{s c}+\tau_{c a}$.

\section{Networked Controller}

The networked controller consists of two parts: a speed controller and a state predictor. A fuzzy logic PI controller is employed as the speed controller, where the gain values are tuned online by the fuzzy logic mechanism. The state predictor is designed in the feedback channel to compensate the negative impact brought by the feedback delay $\tau_{s c}$.

\section{Actuator}

The actuator is triggered when receiving data from the controller. The buffer size of the actuator is 1 , to guarantee the latest control packet is used.

\section{E. Local Controller}

The local controller consists of the current regulator and the flux observer. A sliding mode estimator and a PI controller are adopted as the flux observer and current regulator, respectively. For more details, the readers can refer to [11] and the references therein. Using field orientation technique, the induction motor model is simplified as a DC motor linear model. The synchronous rotating angle of the rotor flux can be calculated from the estimated flux:

$$
\hat{\theta}_{e}=\arctan \left(\hat{\psi}_{\beta r} / \hat{\psi}_{\alpha r}\right) \text {. }
$$


The stator currents under the synchronous rotating $d-q$ coordination are obtained by

$$
\left[\begin{array}{c}
i_{d s} \\
i_{q s}
\end{array}\right]=\left[\begin{array}{cc}
\cos \left(\hat{\theta}_{e}\right) & \sin \left(\hat{\theta}_{e}\right) \\
-\sin \left(\hat{\theta}_{e}\right) & \cos \left(\hat{\theta}_{e}\right)
\end{array}\right]\left[\begin{array}{c}
i_{\alpha s} \\
i_{\beta s}
\end{array}\right],
$$

and the rotor fluxes $\hat{\psi}_{q r}=0$ and $\hat{\psi}_{d r}=\sqrt{\hat{\psi}_{\alpha r}^{2}+\hat{\psi}_{\beta r}^{2}}$ are satisfied under rotor field orientation. Accordingly, the mechanical equation (1e) can be represented as

$$
\dot{\omega}=\frac{K_{t}}{J} i_{q s}-\frac{K_{f}}{J} \omega-\frac{T_{L}}{J},
$$

where $K_{t}=\mu \hat{\psi}_{d r}$

\section{Networked Speed Controller Design}

In this section, the rational gain $K_{P}$ of the state feedback controller is determined using the Lyapunov method. For a single control loop within the NMCS, the mechanical equation (5) can be written in the form of the state space equation:

$$
\left\{\begin{array}{l}
\dot{\bar{x}}(t)=A \bar{x}(t)+B u(t)+E \\
y(t)=C \bar{x}(t)
\end{array}\right.
$$

where $\bar{x}(t)=\omega(t), u(t)=i_{q s}(t), E=-\frac{T_{L}}{J}$, and $y(t)=\omega(t)$, with the coefficients of $A=-\frac{K_{f}}{J}, B=\frac{K_{t}}{J}$, and $C=1$. Taking into account that (6a) can be represented as

$$
\frac{\mathrm{d}}{\mathrm{d} t}\left(\bar{x}+\frac{E}{A}\right)=A\left(\bar{x}+\frac{E}{A}\right)+B u(t),
$$

the closed loop NMCS model can be expressed as

$$
\left\{\begin{array}{l}
\dot{x}(t)=A x(t)+B u(t) \\
y(t)=C x(t)
\end{array},\right.
$$

in stability analysis for convenience.

The maximum allowed time delay $\bar{\tau}$ is predetermined for a specific network protocol. Substituting the state feedback controller $u(t)=-K_{P} x(t-\tau)$ into the closed-loop NMCS model (8), the following equation is held:

$$
\dot{x}(t)=A x(t)+M x(t-\tau),
$$

with $M=-B K_{P}$. Several criteria are introduced to analyze the upper allowed limit of $K_{P}$ :

Lemma 1: [12] Assume that $a(\cdot) \in \mathbb{R}^{n_{a}}, b(\cdot) \in \mathbb{R}^{n_{b}}$, and $W(\cdot) \in \mathbb{R}^{n_{a} \times n_{b}}$ are defined on the interval $\Omega$. For any matrices $X \in \mathbb{R}^{n_{s} \times n_{s}}, Y \in \mathbb{R}^{n_{s} \times n_{b}}$, and $Z \in \mathbb{R}^{n_{b} \times n_{b}}$ satisfying $\left[\begin{array}{cc}X & Y \\ Y^{T} & Z\end{array}\right] \geq 0$, the following inequality holds:

$$
\begin{aligned}
& -2 \int_{\Omega} a^{\mathrm{T}}(\alpha) W b(\alpha) \mathrm{d} \alpha \\
& \quad \leq \int_{\Omega}\left[\begin{array}{l}
a(\alpha) \\
b(\alpha)
\end{array}\right]^{\mathrm{T}}\left[\begin{array}{cc}
X & Y-W \\
Y^{\mathrm{T}}-W^{\mathrm{T}} & Z
\end{array}\right]\left[\begin{array}{l}
a(\alpha) \\
b(\alpha)
\end{array}\right] \mathrm{d} \alpha .
\end{aligned}
$$

The Schur complement lemma can be transformed into the form of Riccati inequality:

Lemma 2: [13] For the given constant matrices $\mathscr{A}$ and $\mathscr{Q}=\mathscr{Q}^{\mathrm{T}}$, if exists matrix variable $\mathscr{P}>0$ satisfying

$$
\left[\begin{array}{cc}
\mathscr{Q} & \mathscr{A} \\
\mathscr{A}^{\mathrm{T}} & -\mathscr{P}^{-1}
\end{array}\right]<0
$$

then the following inequality holds:

$$
\mathscr{A} \mathscr{P} \mathscr{A}^{\mathrm{T}}+\mathscr{Q}<0 \text {. }
$$

The following theorem represents the delay-dependent stability condition of the NMCS:

Theorem 1: If there exist matrices $P>0, Q>0$, and $X$, $Y, Z$ with appropriate dimensions such that

$$
\left[\begin{array}{ccc}
\Gamma & P M-Y & \bar{\tau} A Z \\
M^{\mathrm{T}} P^{\mathrm{T}}-Y^{\mathrm{T}} & -Q & \bar{\tau} M Z \\
\bar{\tau} Z^{\mathrm{T}} A^{\mathrm{T}} & \bar{\tau} Z^{\mathrm{T}} M^{\mathrm{T}} & -\bar{\tau} Z
\end{array}\right]<0,
$$

and

$$
\left[\begin{array}{cc}
X & Y \\
Y^{\mathrm{T}} & Z
\end{array}\right] \geq 0
$$

where $\Gamma=A^{\mathrm{T}} P+P A+Y+Y^{\mathrm{T}}+Q+\bar{\tau} X$, then the system (9) is asymptotically stable for any time delay $0 \leq \tau \leq \bar{\tau}$.

Using Theorem 1, the rational range of the networked speed controller gain in each control loop can be obtained via the given $\bar{\tau}$. For $\bar{\tau}$ is normally a determined value under different network conditions, Theorem 1 gives the reference to set the original value of the controller gain.

Considering the influence of the QoS variation on the control performance, fuzzy logic is adopted in gain adaptation of the networked speed controller. Furthermore, a state predictor placed is employed to minimize the trajectory deviation due to the time delay. In the fuzzy logic tuned networked speed controller, the updating law of the gains are

$$
\begin{aligned}
K_{P}^{\prime} & =K_{P}+\Delta K_{P} \\
K_{I}^{\prime} & =K_{I}+\Delta K_{I}
\end{aligned},
$$

where $\Delta K_{P}$ and $\Delta K_{I}$ are the increment values of $K_{P}$ and $K_{I}$, respectively, while $K_{P}^{\prime}$ and $K_{I}^{\prime}$ are the updated gains. The initial value of $K_{P}$ should take the reference of Theorem 1, and the initial value of $K_{I}$ is given by a small constant.

The state predictor is used to compensate $\tau_{s c}$, to obtain a more accurate plant state estimation. Considering $K_{f}$ is very little when the induction motor running in the constant power region, (5) can be expressed as

$$
\dot{\omega}=\frac{K_{t}}{J} i_{q s}-\frac{T_{L}}{J},
$$

therefore the motor speed can be obtained by

$$
\omega(t)=\omega\left(t_{0}\right)+\frac{K_{t}}{J} \int_{t_{0}}^{t} i_{q s}(s) \mathrm{d} s-\frac{T_{L}}{J}\left(t-t_{0}\right) .
$$

The compensated speed signal within $[k h,(k+1) h]$ can be represented by the following discretized equation:

$$
\hat{\omega}\left(k h+\tau_{s c, k}\right)=\omega(k h)+\left(\frac{K_{t}}{J} i_{q s}(k h)-\frac{1}{J} T_{L}\right) \tau_{s c, k} .
$$

\section{INTEGRATED FEEDBACK SCHEDULING}

In the proposed integrated feedback scheduling method, the sampling period and priority of each control loop is allocated under the constraints of stability and available network bandwidth, to realize the global optimization of the NMCS performance. The speed coupling error $e^{*}(t)$ is also calculated as a reference in calculating the control 
law, therefore, the motion coordination of multiple controlled induction motors is achieved. Denote the assigned bandwidth to the control loop $i$ by $b_{i}=c_{i} / h_{i}$, where $c_{i}$ and $h_{i}$ are the data processing time and sampling period, respectively. The schedulability criterion can refer the sufficient condition in applying the RM scheduling strategy in a general NCS:

Lemma 3: [8] For a NCS with $N$ independent control loops, where a non-preemptive control network is used, the NCS is schedulable with RM algorithm if (19) is satisfied for $i=1, \cdots, N$ :

$$
b_{1}+b_{2}+\ldots+b_{i}+\bar{c}_{i} / h_{i} \leq i\left(2^{1 / i}-1\right),
$$

where $h_{1} \leq h_{1} \leq \ldots h_{n} ; \bar{c}_{i}$ is the worst-case blocking time of task $i$ by lower priority tasks, i.e., $\bar{c}_{i}=\max _{j=i+1, \ldots, N} c_{j}$

\section{A. Optimal Sampling Period Assignment}

The optimal sampling period assignment policy is presented based on minimizing the transmission error between two contiguous sampling periods, with the constraints of stability and communications. The policy can be called the optimal bandwidth allocation (OBA) method. The bandwidth allocation problem can be formulated as a generic constrained optimization problem, which is shown in equations below:

$$
\begin{aligned}
& \text { Minimize }: J\left(h_{i}\right)=\sum_{i=1}^{N} J_{i}\left(h_{i}\right), \\
& \text { Subject to }: 0 \leq h_{i} \leq \bar{\tau}_{i}, \\
& \qquad b_{1}+b_{2}+\ldots+b_{i}+\bar{c}_{i} / h_{i} \leq i\left(2^{1 / i}-1\right),
\end{aligned}
$$

where $J_{i}\left(h_{i}\right)$ is the QoC of loop $i$, and (20b) and (20c) are the stability constraint and schedulability constraint, respectively. Consider the closed-loop model of loop $i$ :

$$
\left\{\begin{array}{l}
\dot{x}_{i}(t)=A_{i} x_{i}(t)+B_{i} u_{i}(t) \\
y_{i}(t)=C_{i} x_{i}(t)
\end{array},\right.
$$

where $x_{i}(t)=y_{i}(t)=\omega_{i}(t), u_{i}(t)=i_{i}^{q s}(t), A_{i}=-D_{i} / J_{i}, B_{i}=$ $K_{i}^{t} / J_{i}, C_{i}=1$, the subscript $i$ denotes the parameters in loop $i$. Substituting the feedback control law $u_{i}(t)=-K_{i}^{P} x_{i}(k h)$ into (21), the following equation is generated:

$$
\dot{x}_{i}(t)=A_{i} x(t)+M_{i} x_{i}(k h),
$$

where $M_{i}=B_{i} K_{i}^{P}$. The state transmission error is defined as the error in the arrived interval of two contiguous control law package:

$$
d_{i}(t)=x_{i}(t)-x_{i}\left(t_{k}\right)
$$

with the dynamics of

$$
\begin{aligned}
\dot{d}_{i}(t)=\dot{x}_{i}(t) & =A_{i} x_{i}(t)-M_{i} x_{i}(k h) \\
& =A_{i}\left(d_{i}(t)+x_{i}(k h)\right)-M_{i} x_{i}(k h) \\
& =A_{i} d_{i}(t)+\left(A_{i}-M_{i}\right) x_{i}(k h) .
\end{aligned}
$$

By solving the first order linear differential equation (24), the Euclidean norm of the ratio between transmitted error and transmitted data can be obtained:

$$
\left\|\frac{d_{i}(t)}{x_{i}(k h)}\right\|=\frac{\left(A_{i}-M_{i}\right)}{A_{i}}\left(1-e^{A_{i} t}\right) .
$$

Therefore, the performance cost function is defined as

$$
J_{i}\left(h_{i}\right)=\frac{\left(A_{i}-M_{i}\right)}{A_{i}}\left(1-e^{A_{i} h_{i}}\right) .
$$

For $A_{i}<0, J_{i}\left(h_{i}\right)$ is a monotonically increasing function, resulting in the maximization of the QoC can be formulated as maximizing (26) with constraints.

\section{B. Optimal Sampling Period Assignment}

In priority-driven network protocols, the control loop with higher data transmission priority has short time delay and lower packet dropouts rate. In the proposed scheduling method, the higher priority is dynamically assigned to the control loop that more urgently needs to send the message. The key issue of the online priority modification (OPM) method is to assign priorities as a function of the errors obtained from the remote controlled plants. The control loop with larger errors would be assigned with the higher priority. The criterion of assigning priorities is the absolute value of the feedback speed error at each sampling instant:

$$
J_{i}^{\prime}(k)=\left|e_{i}(k)\right|,
$$

where $e_{i}(k)=\omega_{i}^{*}(k)-\omega_{i}(k)$, with $\omega_{i}^{*}(k)$ is the reference speed of loop $i$ at the $k^{\text {th }}$ sampling instant. Since $J_{i}^{\prime}$ varies over time, a threshold $\delta$ is introduced to reduce the unnecessary priorities switching caused by small variations of QoC. The rules of the OPM method are listed as following:

1) If $\max \left\{J^{\prime}{ }_{i}\right\}-\min \left\{J^{\prime}{ }_{i}\right\} \leq \delta$ is held for $i=1, \cdots, N$, then keep the current priorities order;

2) If $\left|J^{\prime}{ }_{i}(k)-J^{\prime}{ }_{i}(k-1)\right| \leq \delta$ is held for $i=1, \cdots, N$, then keep the current priorities order;

3) If $\left|J^{\prime}{ }_{i}(k)-J^{\prime}{ }_{j}(k)\right| \leq \delta$ is held, then keep the current priorities order for loop $i$ and loop $j$;

4) If $J^{\prime}{ }_{i}(k)-J^{\prime}{ }_{j}(k)>\delta$ is held, then $p_{i}(k)>p_{j}(k)$.

The procedure of the scheduling and control co-design is shown as Algorithm 1. The performance of the proposed optimal bandwidth scheduling and online priority modification schemes are evaluated by the integral of absolute speed tracking errors (IASTE):

$$
\text { IASTE }=\sum_{i=1}^{N} \int_{0}^{\infty}\left|e_{i}(t)\right| d t .
$$

\section{Simulation Results}

To verify the proposed co-design procedure and demonstrate its effectiveness, simulation studies are carried out for a NMCS including 4 control loops using the TrueTime toolbox on MATLAB/Simulink. The network type is CSMA/AMP (CAN), the data rate is $80 \mathrm{~K}$ bits/s, and the minimum frame size is 32 bits. The reference speed of the induction motors are set as an identical value of $\omega^{*}=100 \mathrm{rad} / \mathrm{s}$. Parameters of the 4 motors in simulation are listed in Table I:

Simulation results are done under two typical QoS conditions: (1) short and constant transmission time $\left(\tau_{i}=2 \mathrm{~ms}\right)$; (2) long and time-varying transmission time (2 ms $\leq \tau_{\mathrm{i}} \leq$ $4 \mathrm{~ms}$ ). Substituting $\bar{\tau}$ into Theorem 1, the obtained upper 


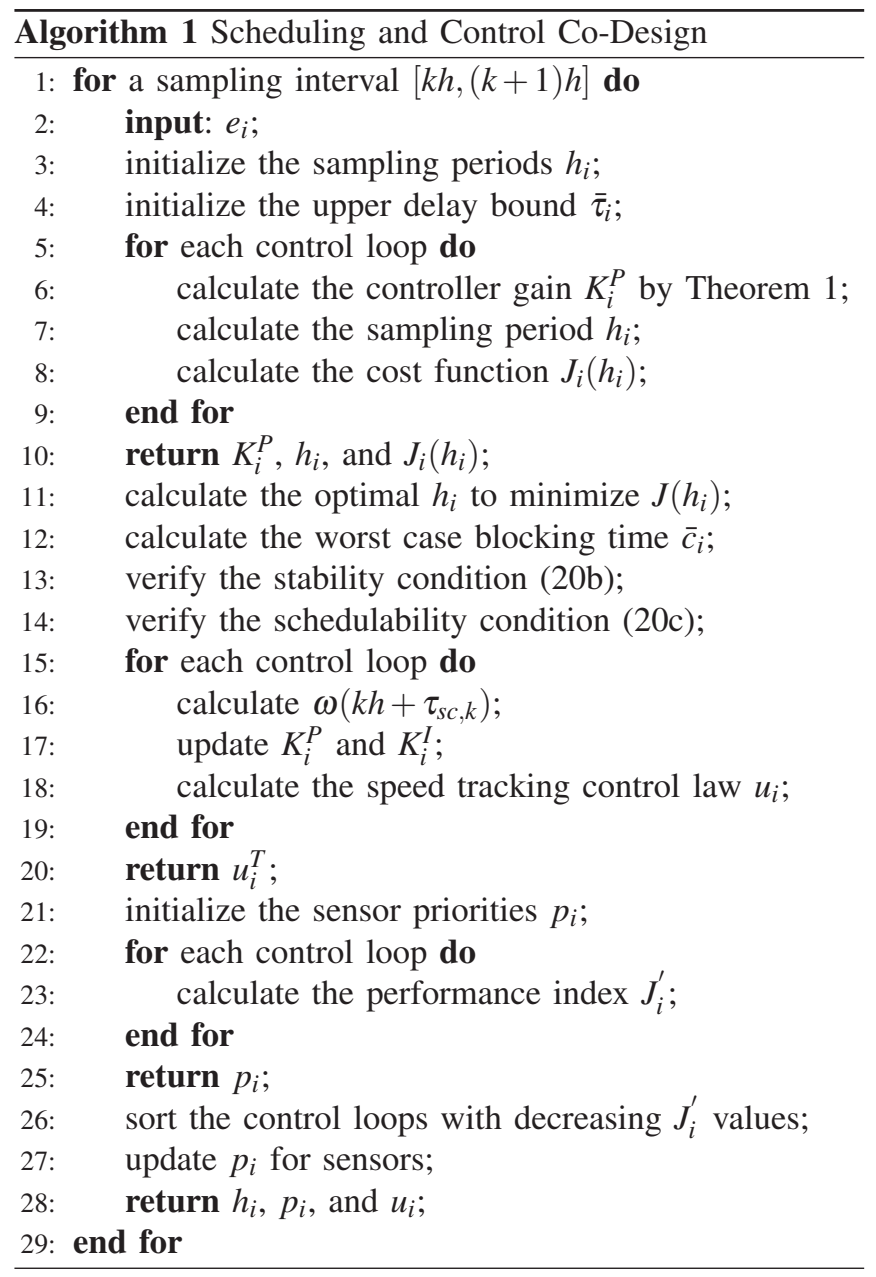

TABLE I

PARAMETERS OF INDUCTION MOTORS

\begin{tabular}{lllll}
\hline Parameters & Motor 1 & Motor 2 & Motor 3 & Motor 4 \\
\hline$R_{S} / \Omega$ & 6.700 & 5.460 & 3.670 & 8.000 \\
$R_{r} / \Omega$ & 5.500 & 4.450 & 2.100 & 3.600 \\
$L_{s} / \mathrm{H}$ & 0.475 & 0.492 & 0.245 & 0.470 \\
$L_{r} / \mathrm{H}$ & 0.475 & 0.492 & 0.245 & 0.470 \\
$M / \mathrm{H}$ & 0.450 & 0.475 & 0.224 & 0.450 \\
$J /\left(\mathrm{kgm}^{2}\right)$ & 0.015 & 0.015 & 0.016 & 0.015 \\
$\psi_{r}^{*} / \mathrm{Wb}$ & 1.000 & 1.000 & 1.000 & 1.000 \\
\hline
\end{tabular}

allowed feedback gains of the four control loops are shown in Table II.

The simulation studies are conducted in 4 different cases, which are

1) The comparison of the proposed OBA scheme with the fixed bandwidth allocation (FBA) scheme.

2) The comparison of the OPM scheme with the fixed priority assignment (FPA) scheme.

3) The comparison of the fuzzy logic speed controller with the memoryless state feedback speed controller.

4) Performance evaluation of the state predictor in time delay compensation.

The simulation results are illustrated in the following.

Case 1. In the FBA scheme, the sampling period of each loop is selected as identical. Under the two QoS conditions,
TABLE II

UPPER LiMITS OF THE FEEDBACK GAINS

\begin{tabular}{lllll}
\hline QoS condition & $\bar{K}_{1}^{P}$ & $\bar{K}_{2}^{P}$ & $\bar{K}_{3}^{P}$ & $\bar{K}_{4}^{P}$ \\
\hline Condition 1 & 4.0 & 4.0 & 2.5 & 4.0 \\
Condition 2 & 2.0 & 2.0 & 1.5 & 2.0 \\
\hline
\end{tabular}

the sampling period is selected as $h_{i}=0.02 \mathrm{~s}$ and $h_{i}=0.03 \mathrm{~s}$, respectively. In the OBA scheme, the optimization problem can be solved using the MATLAB function fmincon, and the optimized sampling period of all the loops are listed in Table III. The simulation results are demonstrated in Fig. 3, where the IASTE are reduced under both of the test conditions using the proposed OBA scheme.

TABLE III

The Optimal SAMPLing Period of EACH LoOP

\begin{tabular}{lllll}
\hline QoS condition & $h_{1}$ & $h_{2}$ & $h_{3}$ & $h_{4}$ \\
\hline Condition 1 & 0.010 & 0.010 & 0.011 & 0.011 \\
Condition 2 & 0.020 & 0.020 & 0.022 & 0.022 \\
\hline
\end{tabular}

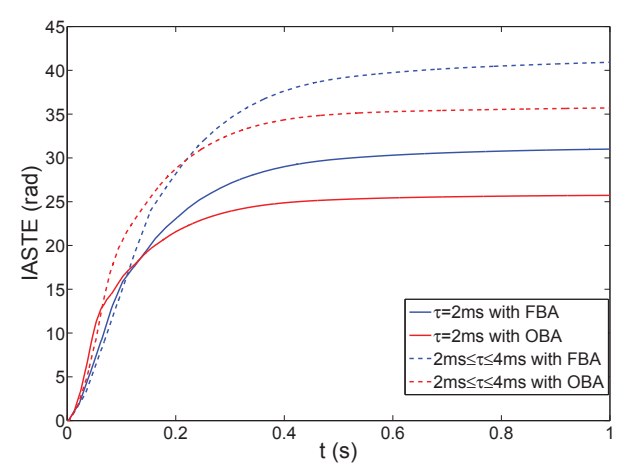

Fig. 3. Comparison of the FBA and OBA

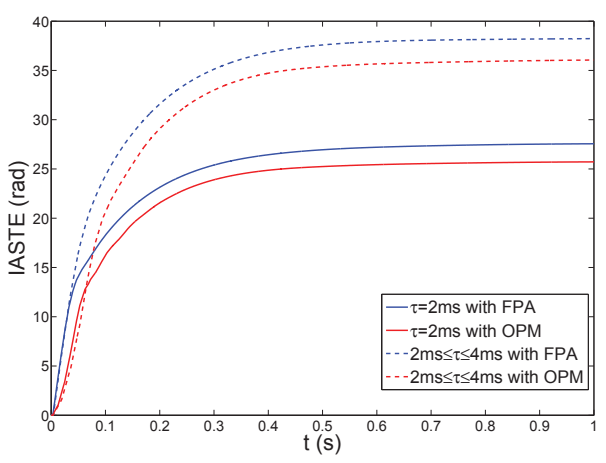

Fig. 4. Comparison of the FPA and OPM

Case 2. The comparison of the OPM scheme with the FPA scheme is shown in Fig. 4. In the FPA scheme, the initial priority of each loop is identical to its index $(1,2, \cdots, N)$. The simulation results show that the IASTE with OPM is less than that with FPA under both test conditions, which showed the effectiveness of the proposed scheduling method. For the 
OPM method is applied on the application layer, modification on the network MAC protocol is not required.

Case 3. The comparison of different networked controllers is presented in Fig. 5. The system performance using a $\mathrm{P}$ controller has the slowest response and largest steady error. This is reasonable since it uses the least information about the system. By employing a PI controller, the steady error of the NCS is improved, but the dynamic response still cannot meet our requirement. However, using the proposed fuzzy logic tuning PI controller, the dynamic response is fast and the steady error is much smaller than the above two controllers. This is because the fuzzy PI controller can tune its gains adaptively according to the output speed and the QoS.
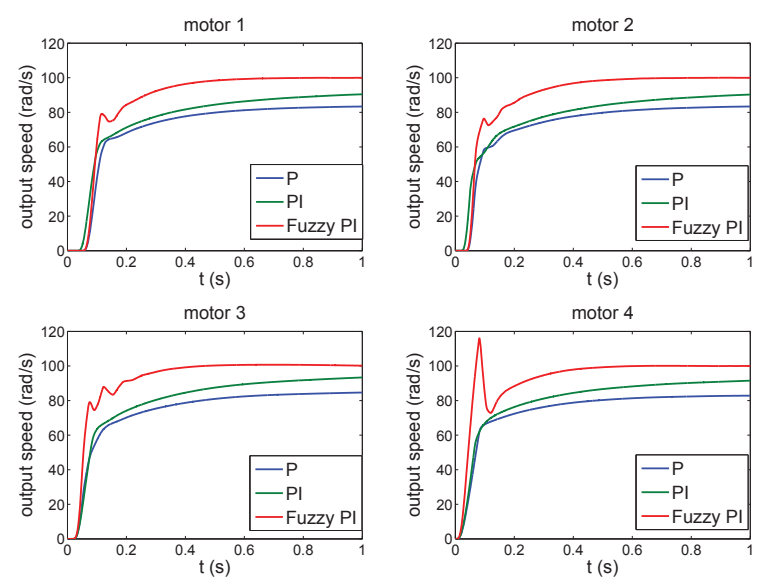

Fig. 5. Comparison of networked controllers in condition 2
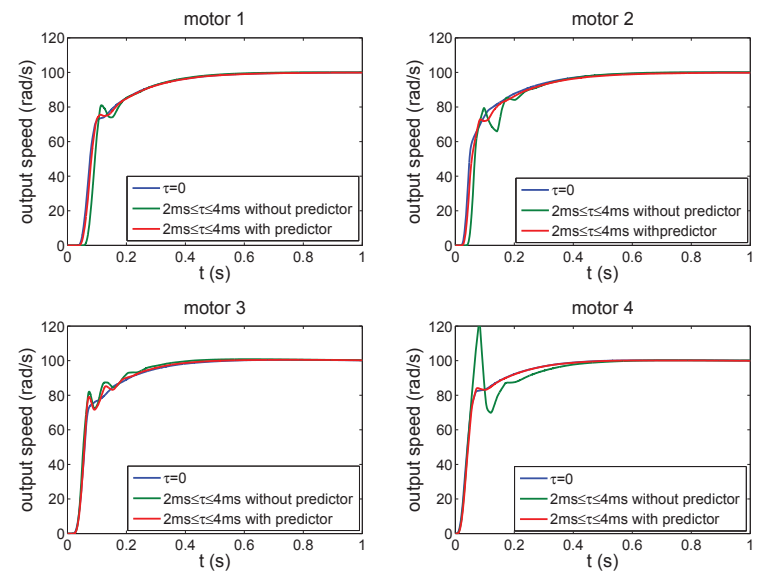

Fig. 6. Performance evaluation of the state predictor in condition 2

Case 4. The performance evaluation of the state predictor under the appointed two conditions is illustrated in Fig. 6. When the NMCS suffers no time delay, the speed tracking performance is the best. When $\tau$ is induced and without delay compensation, the system performance deteriorated. With the state predictor applied, the speed tracking performance is improved, especially on the oscillation.

\section{CONCLUSIONS}

In this paper, an integrated feedback scheduling strategy is proposed for motion coordination operation of multiple induction motors via a shared control network, and its codesign with a networked speed controller was developed. The scheduling strategy includes the optimal bandwidth allocation scheme and online priority modification scheme. The optimal bandwidth allocation scheme minimized the transmission errors, satisfying the stability constraint and the schedulability constraint. The online priority modification scheme decided the data transmission order by sorting the real-time speed feedback errors, therefore the control loops can send their data packet according to their urgency level. The upper limit of the gain of the static feedback networked speed controller is calculated employing the Lyapunov theorem. Furthermore, the closed-loop control performance was improved by online tuning of the gains, together with a state predictor in the feedback channel. Simulation results were conducted in several cases and demonstrated the effectiveness of the co-design methodology under constant delay and time-variable delay, respectively.

\section{REFERENCES}

[1] H. Wu, L. Lou, C. C. Chen, S. Hirche, and K. Kuhnlenz, "Cloud-based networked visual servo control," IEEE Transactions on Industrial Electronics, vol. 60, no. 2, pp. 554-566, 2013.

[2] J. Ferruz, V. M. Vega, A. Ollero, and V. Blanco, "Reconfigurable control architecture for distributed systems in the hero autonomous helicopter," IEEE Transactions on Industrial Electronics, vol. 58, no. 12, pp. 5311-5318, 2011.

[3] H. J. Cho, H. Son, D. G. Lee, T. Bhattacharjee, and D. Y. Lee, "Gain-scheduling control of teleoperation systems interacting with soft tissues," IEEE Transactions on Industrial Electronics, vol. 60, no. 3, pp. 946-957, 2013.

[4] B. K. Kim, W. K. Chung, and I. H. Suh, "Robust synchronizing motion control of twin-servo systems based on network modeling," in the $39^{\text {th }}$ IEEE Conference on Decision and Control, 2000, pp. 1019-1024.

[5] H. B. Li, M. Y. Chow, and Z. Q. Sun, "EDA-based speed control of a networked dc motor system with time delays and packet losses," IEEE Transactions on Industrial Electronics, vol. 56, no. 5, pp. 1727-1735, 2009.

[6] M. Donkers, W. P. M. H. Heemels, N. van de Wouw, and L. Hetel, "Stability analysis of networked control systems using a switched linear systems approach," IEEE Transactions on Automatic Control, vol. 56, no. 9, pp. 2101-2115, 2011.

[7] H. Zhang, Y. Shi, and M. X. Liu, " $H_{\infty}$ step tracking control for networked discrete-time nonlinear systems with integral and predictive actions," IEEE Transactions on Industrial Informatics, vol. 9, no. 1, pp. 337-345, 2013.

[8] C. L. Liu and J. W. Layland, "Scheduling algorithms for multiprogramming in a hard-real-time environment," Journal of the ACM, vol. 20 , no. 1 , pp. 46-63, 1973.

[9] S. L. Dai, H. Lin, and S. S. Ge, "Scheduling-and-control codesign for a collection of networked control systems with uncertain delays," IEEE Transactions on Control Systems Technology, vol. 18, no. 1, 2010.

[10] B. K. Bose, Modern Power Electronics and AC Drives. New Jersey, USA: Prentice Hall, 2002.

[11] Z. Yan and V. Utkin, "Sliding mode observers for electric machines - An overview," in Proceedings of the 28th Annual Conference of the IEEE Industrial Electronics Society, 2002, pp. 1842-1847.

[12] P. Park, Y. S. Moon, and W. H. Kwon, "A delay-dependent robust stability criterion for uncertain time-delay systems," in the 1998 American Control Conference, 1998, pp. 1963-1964.

[13] S. Boyd, L. E. Ghaoui, E. Feron, and V. Balakrishnan, Linear Matrix Inequalities in System and Control Theory. Philadelphia, USA: PA of SIAM, 1994. 